Bull. Korean Math. Soc. 50 (2013), No. 5, pp. 1481-1494

http://dx.doi.org/10.4134/BKMS.2013.50.5.1481

\title{
SOME PROPERTIES OF EVOLUTION ALGEBRAS
}

\author{
L. M. Camacho, J. R. Gómez, B. A. Omirov, and R. M. Turdibaev
}

\begin{abstract}
The paper is devoted to the study of finite dimensional complex evolution algebras. The class of evolution algebras isomorphic to evolution algebras with Jordan form matrices is described. For finite dimensional complex evolution algebras the criterium of nilpotency is established in terms of the properties of corresponding matrices. Moreover, it is proved that for nilpotent $n$-dimensional complex evolution algebras the possible maximal nilpotency index is $1+2^{n-1}$.
\end{abstract}

\section{Introduction}

In 20 s and 30 s of the last century a new object was introduced to mathematics, which was the product of interactions between Mendelian genetics and mathematics. Mendel established the basic laws for inheritance, which are summarized as Mendel's Law of Segregation and Mendel's Law of Independent Assortment. This laws were mathematically formulated by Serebrowsky [10], who was also the first to give an algebraic interpretation of the " $\times$ " sign, which indicated sexual reproduction. Later Glivenkov [6] used the notion of Mendelian algebras in his work. Also Kostitzin [7] independently introduced a "symbolic multiplication" to express Mendel's laws. In his several papers Etherington [3]-[5] introduced the formal language of abstract algebra to the study of genetics. These algebras, in general, are non-associative.

However, in the beginning of the XX century in genetics there were discovered several examples of inheritances, where traits do not segregate in accordance with Mendel's laws. In the present day, non-Mendelian genetics is a basic language of molecular genetics. Non-Mendelian inheritance plays an important role in several disease processes. Naturally, the question arises: What nonMendelian genetics offers to mathematics? The evolution algebras, introduced in [12] serve as the answer to this question.

The concept of evolution algebras lies between algebras and dynamical systems. Algebraically, evolution algebras are non-associative Banach algebra; dynamically, they represent discrete dynamical systems. Evolution algebras

Received October 7, 2011; Revised May 6, 2012.

2010 Mathematics Subject Classification. 05C25, 17A36, 17D92, 17D99.

Key words and phrases. evolution algebra, commutative algebra, isomorphism, nilpotency. 
have many connections with various branches of mathematics, such as graph theory, group theory, stochastic processes, mathematical physics etc. Since evolution algebras are not defined by identities, they can not belong to any well-known classes of non-associative algebras, as Lie, alternative and Jordan algebras. In recent years several authors have tried to investigate the evolution algebras (see [1], [2], [12]).

The foundation of evolution algebra theory and applications in non-Mendelian genetics and Markov chains are developed, with pointers to some further research topics in book [11].

In genetic, the action of genes is manifested statistically in sufficiently large communities of matching individuals (belonging to the same species). These communities are called populations [8]. The population exists not only in space but also in time, i.e., it has its own life cycle. The basis for this phenomenon is reproduction by mating. Mating in a population can be free or subject to certain restrictions.

The evolution of a population comprises a determined change of state in the next generations as a result of reproduction and selection. This evolution can be studied by using the properties of the evolution algebras. It is possible to characterize the disappearance of a population by means of the nilpotency and solvability of the algebra. More precisely, we identify the multiplication of algebra as the reproduction of the species. Hence the solvability is the reproduction of the same generations and the nilpotency is the reproduction of the species but not necessarily of the same generation. Both concepts allow us to study the disappearance of a population.

In this paper, we study some properties of finite dimensional complex evolution algebras. Since any evolution algebra in a natural basis is defined by a quadratic matrix, we study the connection between the algebraic structure of evolution algebras and matrices. More precise results are obtained for evolution algebras with non-singular matrices. For example, the only automorphisms for such algebras are the composition of basis permutation and the multiplication of basic vectors by scalars. Since in the matrix theory the Jordan form of the matrix is essential topic, we investigate a class of evolution algebras isomorphic to evolution algebras with Jordan form matrices. Thus we can distinguish the class of evolution algebras with a matrix in which the eigenvalues are known. Therefore, corresponding algebras can be investigated by the eigenvalues from an algebraical point of view. Namely, the problem of reconstruction of Markov chains on trees [9] which depends on the second eigenvalue can be studied by above evolution algebras.

In [5] it was pointed out for general genetic algebras that the nilpotent property is essential to these algebras and the definition as train algebras and baric algebras were formulated. By this means, we define nil, solvable, rightnilpotent and nilpotent evolution algebras as in [1] and study some properties of $n$-dimensional nilpotent evolution algebras. The notions as right nilpotency and nility for finite dimensional evolution algebras are equivalent [1]. In this work, 
we prove that any $n$-dimensional right-nilpotent evolution algebra is nilpotent. Moreover, for evolution algebras of dimension $n$ we describe some possible values for indexes of nilpotency and prove that $1+2^{n-1}$ is a maximal nilpotency index.

\section{Preliminaries}

Now we define the main object of the paper.

Definition 2.1 ([11]). Let $E$ be a vector space over a field $K$ with defined multiplication $\cdot$ and a basis $\left\{e_{1}, e_{2}, \ldots\right\}$ such that

$$
\begin{gathered}
e_{i} \cdot e_{j}=0, i \neq j, \\
e_{i} \cdot e_{i}=\sum_{k} a_{i k} e_{k}, i \geq 1,
\end{gathered}
$$

then $E$ is called an evolution algebra and basis $\left\{e_{1}, e_{2}, \ldots\right\}$ is said to be natural basis.

From the above definition it follows that evolution algebras are commutative.

Let $E$ be a finite dimensional evolution algebra with natural basis $\left\{e_{1}, \ldots\right.$, $\left.e_{n}\right\}$, then

$$
e_{i} \cdot e_{i}=\sum_{j=1}^{n} a_{i j} e_{j}, 1 \leq i \leq n
$$

where remaining products are equal to zero.

The matrix $A=\left(a_{i j}\right)$ with $1 \leq i, j \leq n$ is called matrix of the algebra $E$ in natural basis $\left\{e_{1}, \ldots, e_{n}\right\}$.

In [11] conditions for basis transformations that preserve naturalness of the basis are given. Also, the relation between the matrices in a new and old natural basis is established in terms of new defined operation on matrices. Since this approach is not practical for our further purposes, below we give the following brief version in terms of its matrix elements.

Now let us consider non-singular linear transformation of the given natural basis $\left\{e_{1}, \ldots, e_{n}\right\}$ by matrix $T=\left(t_{i j}\right)$ with $1 \leq i, j \leq n$ :

$$
f_{i}=\sum_{j=1}^{n} t_{i j} e_{j}, 1 \leq i \leq n .
$$

This transformation is an isomorphism if and only if $f_{i} \cdot f_{j}=0$ for all $i \neq j$. Thus,

$$
f_{i} \cdot f_{j}=\sum_{p=1}^{n} t_{i p} t_{j p}\left(e_{p} \cdot e_{p}\right)=\sum_{k=1}^{n}\left(\sum_{p=1}^{n} t_{i p} t_{j p} a_{p k}\right) e_{k}=0 .
$$

Hence, if $T$ is an isomorphism, then for $i \neq j$ and $1 \leq k \leq n$ we have

$$
\sum_{p=1}^{n} t_{i p} t_{j p} a_{p k}=0
$$


Observe that

$$
f_{i} \cdot f_{i}=\sum_{p=1}^{n} t_{i p}^{2}\left(e_{p} \cdot e_{p}\right)=\sum_{p=1}^{n} t_{i p}^{2} \sum_{k=1}^{n} a_{p k} e_{k}=\sum_{k=1}^{n}\left(\sum_{p=1}^{n} t_{i p}^{2} a_{p k}\right) e_{k} .
$$

Now let $T_{i j}$ be the elements of matrix $T^{-1}$. Then $e_{k}=\sum_{s=1}^{n} T_{k s} f_{s}$ and

$$
f_{i} \cdot f_{i}=\sum_{k=1}^{n}\left(\sum_{p=1}^{n} t_{i p}^{2} a_{p k}\right) \sum_{s=1}^{n} T_{k s} f_{s}=\sum_{s=1}^{n}\left(\sum_{k=1}^{n} \sum_{p=1}^{n} t_{i p}^{2} a_{p k} T_{k s}\right) f_{s} .
$$

Hence, for the elements of the matrix $B=\left(b_{i s}\right)$ with $1 \leq i, s \leq n$ of the evolution algebra $E$ in natural basis $\left\{f_{1}, \ldots, f_{n}\right\}$ we have

$$
b_{i s}=\sum_{k=1}^{n} \sum_{p=1}^{n} t_{i p}^{2} a_{p k} T_{k s} .
$$

Definition 2.2. An element $a$ of the evolution algebra $E$ is called nil if there exists $n(a) \in \mathbb{N}$ such that $(\ldots(\underbrace{(a \cdot a) \cdot a) \cdot \ldots) \cdot a)}_{n(a) \text { times }}=0$. The evolution algebra $E$ is called nil if any element of the algebra is nil.

We introduce the following sequences:

$$
\begin{gathered}
E^{(1)}=E, E^{(k+1)}=E^{(k)} E^{(k)}, k \geq 1, \\
E^{\langle 1\rangle}=E, E^{\langle k+1\rangle}=E^{\langle k\rangle} E, k \geq 1, \\
E^{1}=E, E^{k}=\sum_{i=1}^{k-1} E^{i} E^{k-i}, k \geq 1 .
\end{gathered}
$$

Note that is not difficult to prove the following inclusions for $k \geq 1$ :

$$
E^{\langle k\rangle} \subseteq E^{k}, E^{(k+1)} \subseteq E^{2^{k}} .
$$

Also, note that since $E$ is a commutative algebra we obtain

$$
E^{k}=\sum_{1 \leq i \leq \frac{k}{2}} E^{i} E^{k-i}
$$

Definition 2.3. An evolution algebra $E$ is called

(i) solvable if there exists $n \in \mathbb{N}$ such that $E^{(n)}=0$;

(ii) right nilpotent if there exists $n \in \mathbb{N}$ such that $E^{\langle n\rangle}=0$;

(iii) nilpotent if there exists $n \in \mathbb{N}$ such that $E^{n}=0$.

The minimal numbers $s, r, n$ such that $E^{(s)}, E^{\langle r\rangle}, E^{n}$ are equal to zero are called indexes of solvability, right nilpotency and nilpotency, respectively.

Observe that if an evolution algebra is nilpotent, then it is right nilpotent and solvable. The following example shows that a solvable evolution algebra is not necessarily a right nilpotent algebra. 
Proposition 2.4. Let $E$ be an $n$-dimensional complex evolution algebra such that $\operatorname{dim} E^{(2)}=1$. Then $E^{(3)}=0$ if and only if $E$ is isomorphic to an evolution algebra with natural basis $\left\{e_{1}, \ldots, e_{n}\right\}$ and $k \in\{1, \ldots, n\}$, with the following multiplication:

$$
e_{i} e_{i}=\lambda_{i}\left(e_{1}+\cdots+e_{k}\right), 1 \leq i \leq n
$$

where $\lambda_{i} \in \mathbb{C}, \sum_{j=1}^{k} \lambda_{j}=0, \sum_{j=1}^{n}\left|\lambda_{j}\right| \neq 0$.

Proof. Since $\operatorname{dim} E^{(2)}=1$ and $E^{(2)}$ is spanned by $e_{i} e_{i}, 1 \leq i \leq n$ we obtain that they are collinear to a non-zero vector $x=a_{1} e_{1}+\cdots+a_{n} e_{n}$. Let us suppose that there exist $k$ elements, $\left\{a_{i_{1}}, \ldots, a_{i_{k}}\right\}$, with $1 \leq k \leq n$ such that all of them are non-zeros. With the suitable natural basis change, one can assume that $x=e_{1}+\cdots+e_{k}$ for some $1 \leq k \leq n$.

Let $e_{i} e_{i}=\lambda_{i} x, 1 \leq i \leq n$ and $\sum_{j=1}^{n}\left|\lambda_{j}\right| \neq 0$. Then $E^{(3)}$ is spanned by

$$
x x=\left(e_{1}+\cdots+e_{k}\right)^{2}=\sum_{j=1}^{k} \lambda_{j} x .
$$

Hence, $E^{(3)}=0$ if and only if $\sum_{j=1}^{k} \lambda_{j}=0$.

The following example is a special case of the above proposition:

Example 2.5. Let $E$ be an evolution algebra with natural basis $\left\{e_{1}, \ldots, e_{n}\right\}$ and the following multiplication:

$$
\begin{aligned}
e_{i} e_{i} & =e_{1}+\cdots+e_{n}, 1 \leq i \leq n-1, \\
e_{n} e_{n} & =(1-n)\left(e_{1}+\cdots+e_{n}\right) .
\end{aligned}
$$

Then $E^{(3)}=0$, but $E^{k}=\left\langle e_{1}+\cdots+e_{n}\right\rangle$ for $k \geq 2$.

Remark 2.6. Actually, the multiplication obtained in Proposition 2.4 can be divided into two disjoint classes. First one, when $\lambda_{i}=0$ for all $1 \leq i \leq k$, then this evolution algebra is nilpotent. The second one is when $\lambda_{i} \neq 0$ for some $1 \leq i \leq k$. Then by natural basis transformation one can assume that $e_{1} e_{1}=e_{1}+\cdots+e_{k}$ and hence, this evolution algebra is not nilpotent.

In [1] the equivalence of right nilpotency and nility for finite dimensional evolution algebras is proved.

Theorem 2.7 ([1]). The following statements are equivalent:

a) The matrix of an evolution algebra $E$ can be transformed by natural basis permutation to

$$
A=\left(\begin{array}{ccccc}
0 & a_{12} & a_{13} & \ldots & a_{1 n} \\
0 & 0 & a_{23} & \ldots & a_{2 n} \\
0 & 0 & 0 & \ldots & a_{3 n} \\
\vdots & \vdots & \vdots & \ddots & \vdots \\
0 & 0 & 0 & \ldots & 0
\end{array}\right)
$$


b) Evolution algebra $E$ is right nilpotent algebra;

c) Evolution algebra $E$ is nil algebra.

\section{Isomorphisms}

In case of evolution algebras with non-singular evolution matrices the problem of finding isomorphic algebras to the given one can be solved more precisely.

Let $E$ be an evolution algebra with matrix $A$ such that $\operatorname{det} A \neq 0$.

Proposition 3.1. If we define $T_{\pi}=\left(t_{i j}\right), 1 \leq i, j \leq n$, such that $t_{i j} \neq 0$ if and only if $j=\pi(i)$ with $\pi \in S_{n}$, then $\operatorname{Aut}(E)=\left\{T: T\right.$ is of the form $T_{\pi}$ for some $\left.\pi \in S_{n}\right\}$. Moreover, if $T_{\pi}$ is an automorphism of evolution algebra $E$ and $B=\left(b_{i j}\right)$ with $1 \leq i, j \leq n$ is the matrix of $E$ in basis $T_{\pi}\left(e_{1}\right), \ldots, T_{\pi}\left(e_{n}\right)$, then

$$
b_{i j}=\frac{t_{i, \pi(i)}^{2}}{t_{j, \pi(j)}} \cdot a_{\pi(i) \pi(j)} .
$$

Proof. Let $T$ be an automorphism and $\left(t_{i j}\right)_{1 \leq i, j \leq n}$ the matrix. Consider (2.1) as a linear homogeneous system of equations in terms of unknowns $t_{i 1} t_{j 1}, \ldots$, $t_{i n} t_{j n}$. If $A$ is a non-singular matrix, then from (2.1) we obtain

$$
\left\{\begin{array}{c}
t_{i 1} t_{j 1}=0 \\
t_{i 2} t_{j 2}=0 \\
\cdots \\
t_{i n} t_{j n}=0
\end{array}\right.
$$

where $i \neq j$.

Since matrix $T=\left(t_{i j}\right)$ with $1 \leq i, j \leq n$ is non-singular, in every row there is at least one non-zero element. But for any non-zero element $t_{i p}$ (in the $i$-th row) we have $t_{i p} t_{j p}=0$ for all $j \neq i$. Therefore, $t_{j p}=0$ for $j \neq i$. Now if for some $m \neq p$ we have $t_{i m} \neq 0$, then similarly, we obtain $t_{j m}$ for all $j \neq m$. But this contradicts non-singularity of matrix $T$. Therefore, in every row and every column we have exactly one non-zero element, i.e., the matrix $T$ has the form described in the statement of the proposition.

Note that $\operatorname{det} T=(-1)^{\sigma(\pi)} t_{1 \pi(1)} t_{2 \pi(2)} \cdots t_{n \pi(n)}$, where $\sigma(\pi)$ is the sign of $\pi$.

We obtain that the group of automorphisms of $E$ is $\left\{T_{\pi} \mid \pi \in S_{n}\right\}$ and $T_{\pi} \circ T_{\tau}=T_{\tau \circ \pi}$.

Let us fix one $\pi \in S_{n}$. Then $T\left(e_{i}\right)=t_{i, \pi(i)} e_{\pi(i)}$ for all $1 \leq i \leq n$. Now

$$
\begin{aligned}
T\left(e_{i}\right) \cdot T\left(e_{i}\right) & =t_{i, \pi(i)}^{2}\left(e_{\pi(i)} \cdot e_{\pi(i)}\right)=t_{i, \pi(i)}^{2} \sum_{k=1}^{n} a_{\pi(i) k} e_{k} \\
& =t_{i, \pi(i)}^{2} \sum_{k=1}^{n} a_{\pi(i) \pi(k)} e_{\pi(k)}=\sum_{k=1}^{n} \frac{t_{i, \pi(i)}^{2}}{t_{k, \pi(k)}} a_{\pi(i) \pi(k)} T\left(e_{k}\right) .
\end{aligned}
$$

Hence, the elements of evolution matrix $B=\left(b_{i j}\right)_{1<i, j \leq n}$ of an algebra isomorphic to $E$ satisfy (3.1). 
For a $\pi \in S_{n}$ denote by $s_{\pi}:\{1,2, \ldots, n\} \backslash\left\{\pi^{-1}(n)\right\} \rightarrow\{1,2, \ldots, n\}$ a one-to-one mapping defined by $s_{\pi}(i)=\pi^{-1}(1+\pi(i))$.

Proposition 3.2. Let $A=\left(a_{i j}\right)_{1 \leq i, j \leq n}$ be a matrix of an evolution algebra isomorphic to an evolution algebra with Jordan cell matrix with non-zero eigenvalue $\lambda$. Then the only non-zero elements of $A$ are the diagonal elements and $a_{i, s_{\pi}(i)}$ for all $i \neq \pi^{-1}(n)$ and $\lambda=\frac{a_{i i}^{2}}{a_{i, s_{\pi}(i)} a_{s_{\pi}(i) s_{\pi}(i)}}$ for all $i \neq \pi^{-1}(n)$.

Proof. First consider the isomorphism of evolution algebra with Jordan cell matrix with non-zero eigenvalue $\lambda$. Since the matrix is non-singular, by the proof of Proposition 3.1 we obtain that it is in the form $T_{\pi}$.

For fixed $\pi \in S_{n}$ we put $T_{\pi}\left(e_{i}\right)=f_{i}$ and derive

$$
f_{i} \cdot f_{i}= \begin{cases}t_{i, \pi(i)} \lambda f_{i}+\frac{t_{i, \pi(i)}^{2}}{t_{s_{\pi}(i), \pi\left(s_{\pi}(i)\right)}} f_{s_{\pi}(i)} & \text { for } i \neq \pi^{-1}(n) \\ t_{i, \pi(i)} \lambda f_{i} & \text { for } i=\pi^{-1}(n) .\end{cases}
$$

Hence the matrix of the new evolution algebra is a sum of non-singular diagonal matrix and a matrix that has exactly one non-zero element on each row except the $\pi^{-1}(n)$-th, which is a zero row and at most one non-zero element in each column.

Now let us fix a permutation $\pi \in S_{n}$ and consider matrix $A=\left(a_{i j}\right)_{i, j=1}^{n}$ with zero elements except the diagonal elements and $a_{i, s_{\pi}(i)}$ for all $i \neq \pi^{-1}(n)$ and $s_{\pi}(i)=\pi^{-1}(1+\pi(i))$. If this evolution algebra is isomorphic to an evolution algebra with Jordan cell matrix with eigenvalue $\lambda$, then $a_{i i}=\lambda t_{i, \pi(i)}$ for all $1 \leq i \leq n$ and $a_{i, s_{\pi}(i)}=\frac{t_{i, \pi(i)}^{2}}{t_{s_{\pi}(i), \pi\left(s_{\pi}(i)\right)}}$.

Since $t_{i, \pi(i)}=\frac{1}{\lambda} a_{i i}$ and $t_{s_{\pi}(i), \pi\left(s_{\pi}(i)\right)}=\frac{1}{\lambda} a_{s_{\pi}(i) s_{\pi}(i)}$ we obtain

$$
a_{i, s_{\pi}(i)}=\frac{a_{i i}^{2}}{\lambda^{2}} \cdot \frac{\lambda}{a_{s_{\pi}(i) s_{\pi}(i)}}=\frac{1}{\lambda} \cdot \frac{a_{i i}^{2}}{a_{s_{\pi}(i) s_{\pi}(i)}} \text { and hence } \lambda=\frac{a_{i i}^{2}}{a_{i, s_{\pi}(i)} a_{s_{\pi}(i) s_{\pi}(i)}} \text {. }
$$

Hence, if matrix $A$ satisfies $\lambda=\frac{a_{i i}^{2}}{a_{i, s_{\pi}(i)} a_{s_{\pi}(i) s_{\pi}(i)}}$ for all $i \neq \pi^{-1}(n)$, then evolution algebra with matrix $A$ is isomorphic to evolution algebra with Jordan cell matrix with eigenvalue $\lambda$. This isomorphism has the matrix which is the inverse to $T=\left(t_{i j}\right)_{i, j=1}^{n}$, where $t_{i \pi(i)}=\frac{1}{\lambda} a_{i i}$ and zero otherwise.

The above result can be generalized to the case of Jordan form matrices. Let $J=J_{1} \oplus J_{2} \oplus \cdots \oplus J_{r}$, where $J_{i}$ are Jordan cells of dimension $n_{i}$ with non-zero eigenvalue $\lambda_{i}$.

Now let us denote $\mu_{k}=\lambda_{i}$ for $n_{1}+\cdots+n_{i-1}+1 \leq k \leq n_{1}+\cdots+n_{i}, 1 \leq i \leq r$.

Take $\pi \in S_{n}$ and denote $s_{\pi}^{\prime}:\{1, \ldots, n\} \backslash\left\{\pi^{-1}\left(n_{1}\right), \ldots, \pi^{-1}\left(n_{r}\right)\right\} \rightarrow\{1, \ldots, n\}$ a one-to-one mapping defined by $s_{\pi}^{\prime}(i)=\pi^{-1}(1+\pi(i))$.

Corollary 3.3. Let $A=\left(a_{i j}\right)_{1 \leq i, j \leq n}$ be a matrix of an evolution algebra isomorphic to an evolution algebra with Jordan form matrix J. Then the only 
non-zero elements of $A$ are the diagonal elements and $a_{i, s_{\pi}^{\prime}(i)}$ such that

$$
\frac{a_{i i}^{2}}{a_{i, s_{\pi}^{\prime}(i)} a_{s_{\pi}^{\prime}(i) s_{\pi}^{\prime}(i)}}=\frac{\mu_{i}^{2}}{\mu_{s_{\pi}^{\prime}(i)}}
$$

for $i \notin\left\{\pi^{-1}\left(n_{1}\right), \ldots, \pi^{-1}\left(n_{r}\right)\right\}$.

\section{Nilpotency of evolution algebras}

Let us now consider an evolution algebra $E$ with Jordan cell with eigenvalue $\lambda$.

Proposition 4.1. If $\lambda \neq 0$, then $E$ is neither solvable nor right nilpotent and therefore is not nilpotent.

Proof. Since $\lambda \neq 0$ then evolution matrix is non-degenerated. Therefore, $E^{2}=$ $E^{(2)}=E^{\langle 2\rangle}=E$. By simple induction we obtain $E^{k}=E^{(k)}=E^{\langle k\rangle}=E$ and the statement of the proposition is verified.

Proposition 4.2. For an evolution algebra with Jordan cell matrix and eigenvalue $\lambda=0$ the following statements hold:

(i) $E$ is one generated;

(ii) $E$ is solvable with index of solvability $n+1$;

(iii) $E$ is right nilpotent with index of right nilpotency $n+1$;

(iv) $E$ is nilpotent with index of nilpotency $2^{n-1}+1$.

Proof. (i) From $\lambda=0$ it follows that for basis elements $e_{i}$ of $E$ we have $e_{i} \cdot e_{i}=$ $e_{i+1}$ for all $1 \leq i \leq n-1$ and $e_{n} \cdot e_{n}=0$.

Therefore, $E$ is one-generated: $E=i d\left\langle e_{1}\right\rangle$.

(ii) It follows from $E^{(k)}=\left\langle e_{k}, e_{k+1}, \ldots, e_{n}\right\rangle, 1 \leq k \leq n$ and $E^{(n+1)}=0$.

(iii) It is similar to (ii).

(iv) We claim that

$$
E^{2^{k}+1}=E^{2^{k}+2}=\cdots=E^{2^{k+1}}=\left\langle e_{k+2}, \ldots, e_{n}\right\rangle
$$

for all $0 \leq k \leq n-2$.

Indeed, for $k=0$ we have $E^{2}=E E=\left\langle e_{2}, \ldots, e_{n}\right\rangle$.

For $k=1$ we have

$$
\begin{gathered}
E^{2+1}=E^{3}=E E^{2}=\left\langle e_{3}, \ldots, e_{n}\right\rangle, \\
E^{2^{2}}=E^{4}=E E^{3}+E^{2} E^{2}=\left\langle e_{3}, \ldots, e_{n}\right\rangle .
\end{gathered}
$$

Assume that

$$
E^{2^{k-1}+1}=E^{2^{k-1}+2}=\cdots=E^{2^{k}}=\left\langle e_{k+1}, \ldots, e_{n}\right\rangle .
$$

Using this assumption we obtain

$$
\begin{aligned}
E^{2^{k}+1} & =E E^{2^{k}}+E^{2} E^{2^{k}-1}+\cdots+E^{2^{k-1}} E^{2^{k-1}+1} \\
& =E E^{2^{k}}+E^{2} E^{2^{k}}+\cdots+E^{2^{k-1}} E^{2^{k}}
\end{aligned}
$$




$$
=\left(E+E^{2}+E^{3}+\cdots+E^{2^{k-1}}\right) E^{2^{k}}=E E^{2^{k}}=\left\langle e_{k+2}, \ldots, e_{n}\right\rangle .
$$

Also

$$
\begin{aligned}
E^{2^{k+1}} & =E E^{2^{k+1}-1}+E^{2} E^{2^{k+1}-2}+\cdots+E^{2^{k}} E^{2^{k}} \\
& \supseteq E^{2^{k}} E^{2^{k}}=\left\langle e_{k+2}, \ldots, e_{n}\right\rangle .
\end{aligned}
$$

So we obtain

$$
\left\langle e_{k+2}, \ldots, e_{n}\right\rangle=E^{2^{k}+1} \supseteq E^{2^{k}+2} \supseteq \ldots \supseteq E^{2^{k+1}} \supseteq\left\langle e_{k+2}, \ldots, e_{n}\right\rangle .
$$

Hence,

$$
E^{2^{k}+1}=E^{2^{k}+2}=\cdots=E^{2^{k+1}}=\left\langle e_{k+2}, \ldots, e_{n}\right\rangle .
$$

Therefore, $E^{2^{n-1}}=\left\langle e_{n}\right\rangle$ and $E^{2^{n-1}+1}=0$.

Hence, $E$ is nilpotent with nilpotency index equal to $1+2^{n-1}$.

Remark 4.3. We should note that the statements (ii)-(iv) of Proposition 4.2 are equivalent, since one can show that each of them is equivalent to $\lambda=0$. However, statement (i) is not equivalent to $\lambda=0$ since for $\lambda=1$ one can prove that $E$ is generated by the element $e_{1}+e_{2}$.

Observe that any evolution subalgebra of an evolution algebra is an ideal [11]. Therefore if we consider an evolution algebra $E_{J}$ with matrix $J$ in Jordan form $J=J_{1} \oplus J_{2} \oplus \cdots \oplus J_{r}$, where $J_{i}$ are Jordan cells of dimension $n_{i}$ with eigenvalues $\lambda_{i}$, then

$$
E_{J}=E_{1} \oplus E_{2} \oplus \cdots \oplus E_{r},
$$

where $E_{i}=\left\langle e_{n_{i-1}+1}, \ldots, e_{n_{i}}\right\rangle$.

Now we have $E_{J}^{k}=E_{1}^{k} \oplus E_{2}^{k} \oplus \cdots \oplus E_{r}^{k}$ and therefore $E_{J}$ is nilpotent if and only if every $E_{i}$ is nilpotent. Since we have obtained the criterium of nilpotency of Jordan blocks, we obtain:

Corollary 4.4. $E_{J}$ is nilpotent (with index of nilpotency equal to $\max _{1 \leq i \leq r}\{1+$ $\left.2^{n_{i}-1}\right\}$ ) if and only if $J$ has only zero eigenvalues. The same assertion holds for right nilpotency and solvability with corresponding indexes equal to $1+$ $\max _{1 \leq i \leq r}\left\{n_{i}\right\}$.

Note that from Corollary 4.4 it follows that for every $1 \leq k \leq n$ we obtain an example of nilpotent evolution algebra with index of nilpotency equal to $1+2^{k-1}$

The following theorem represents the criterium of nilpotency of a finite dimensional evolution algebra.

Theorem 4.5. Let $E$ be an n-dimensional evolution algebra. Then $E$ is nilpotent if and only if the matrix of evolution algebra $A$ can be transformed by the natural basis permutation to form (2.3). Moreover, the index of nilpotency of the evolution algebra $E$ is not greater than $2^{n-1}+1$. 
Proof. Let $E$ be a nilpotent. Then it is right nilpotent and therefore, by Theorem 2.7 the matrix of this evolution algebra can be transformed by the natural basis permutation to form (2.3).

Now let the matrix $A$ of $E$ can be transformed by the natural basis permutation to form (2.3).

Assume that $a_{12} a_{23} \cdots a_{n-1 n} \neq 0$. Similar to the proof of (iv) in Proposition 4.2 one can verify

$$
E^{2^{k}+1}=E^{2^{k}+2}=\cdots=E^{2^{k+1}}=\left\langle e_{k+2}, \ldots, e_{n}\right\rangle
$$

for all $0 \leq k \leq n-2$.

Therefore, $E^{2^{n-1}}=\left\langle e_{n}\right\rangle$ and $E^{2^{n-1}+1}=0$.

Hence, $E$ is nilpotent with nilpotency index equal to $1+2^{n-1}$.

Now assume that $a_{12} a_{23} \cdots a_{n-1 n}=0$. In this case we claim that

$$
\left\langle e_{k+2}, \ldots, e_{n}\right\rangle \supseteq E^{2^{k}+1}
$$

for all $0 \leq k \leq n-2$.

Indeed, for $k=0$ we have $E^{2}=E E \subseteq\left\langle e_{2}, \ldots, e_{n}\right\rangle$.

For $k=1$ we have

$$
E^{2+1}=E^{3}=E E^{2} \subseteq\left\langle e_{3}, \ldots, e_{n}\right\rangle .
$$

Assume that

$$
\left\langle e_{k+1}, \ldots, e_{n}\right\rangle \supseteq E^{2^{k-1}+1} .
$$

Using this assumption we obtain

$$
\begin{aligned}
E^{2^{k}+1} & =E E^{2^{k}}+E^{2} E^{2^{k}-1}+\cdots+E^{2^{k-1}} E^{2^{k-1}+1} \\
& \subseteq E E^{2^{k-1}+1}+E^{2} E^{2^{k-1}+1}+\cdots+E^{2^{k-1}} E^{2^{k-1}+1} \\
& =\left(E+E^{2}+E^{3}+\cdots+E^{2^{k-1}}\right) E^{2^{k-1}+1} \subseteq E E^{2^{k-1}+1} \subseteq\left\langle e_{k+2}, \ldots, e_{n}\right\rangle .
\end{aligned}
$$

So we obtain

$$
\left\langle e_{k+2}, \ldots, e_{n}\right\rangle \supseteq E^{2^{k}+1} .
$$

Therefore, $\left\langle e_{n}\right\rangle \supseteq E^{2^{n-2}+1}$.

Hence,

$$
\begin{aligned}
E^{2^{n-1}+1} & =E E^{2^{n-1}}+E^{2} E^{2^{n-1}-1}+\cdots+E^{2^{n-2}} E^{2^{n-2}+1} \\
& \subseteq\left(E+E^{2}+\cdots+E^{2^{n-2}}\right)\left\langle e_{n}\right\rangle \subseteq E\left\langle e_{n}\right\rangle=0 .
\end{aligned}
$$

Thus, $E$ is nilpotent with nilpotency index not greater than $1+2^{n-1}$.

Corollary 4.6. For finite dimensional complex evolution algebra notions as nil, nilpotent and right nilpotent algebras are equivalent. However, the indexes of nility, right nilpotency and nilpotency do not coincide in general.

The following proposition excludes significantly many possible values that a nilpotency indexes of $n$-dimensional evolution algebras can take. 
Proposition 4.7. Let $E$ be a nilpotent evolution algebra with index of nilpotency not equal to $2^{n-1}+1$. Then it is not greater than $2^{n-2}+1$.

Proof. Since $E$ is nilpotent, we assume that the matrix $A$ of $E$ in the natural basis $\left\{e_{1}, \ldots, e_{n}\right\}$ is in the form $(2.3)$.

From the proof of Proposition 4.5 it follows that $a_{12} a_{23} \ldots a_{n-1 n}=0$ and

$$
\left\langle e_{k+2}, \ldots, e_{n}\right\rangle \supseteq E^{2^{k}+1}
$$

for all $0 \leq k \leq n-2$.

Assume that $E$ is nilpotent with index of nilpotency greater than $2^{n-2}+1$ and not equal to $2^{n-1}+1$.

Then $\left\langle e_{n}\right\rangle \supseteq E^{2^{n-2}+1}$ and since $E^{2^{n-2}+1} \neq 0$ we obtain $E^{2^{n-2}+1}=\left\langle e_{n}\right\rangle$.

Therefore, $\left\langle e_{n-1}, e_{n}\right\rangle \supseteq E^{2^{n-3}+1} \supseteq E^{2^{n-3}+2} \supseteq \cdots \supseteq E^{2^{n-2}} \supseteq\left\langle e_{n}\right\rangle$.

Now if $E^{2^{n-3}+1}=E^{2^{n-3}+2}=\cdots=E^{2^{n-2}}=\left\langle e_{n}\right\rangle$, then

$$
\begin{aligned}
E^{2^{n-2}+1} & =E E^{2^{n-2}}+E^{2} E^{2^{n-2}-1}+\cdots+E^{2^{n-3}} E^{2^{n-3}+1} \\
& \subseteq\left(E+E^{2}+\cdots+E^{2^{n-3}}\right)\left\langle e_{n}\right\rangle=E\left\langle e_{n}\right\rangle=0
\end{aligned}
$$

which is a contradiction. Hence, $\left\langle e_{n-1}, e_{n}\right\rangle=E^{2^{n-3}+1}$.

Now assume that $\left\langle e_{n-k}, \ldots, e_{n}\right\rangle=E^{2^{n-k-2}+1}$. Then

$$
\begin{aligned}
\left\langle e_{n-k-1}, e_{n-k}, \ldots, e_{n}\right\rangle & \supseteq E^{2^{n-k-3}+1} \supseteq E^{2^{n-k-3}+2} \\
& \supseteq \cdots \supseteq E^{2^{n-k-2}} \supseteq\left\langle e_{n-k}, \ldots, e_{n}\right\rangle .
\end{aligned}
$$

If $E^{2^{n-k-3}+1} \neq\left\langle e_{n-k-1}, e_{n-k}, \ldots, e_{n}\right\rangle$, then $E^{2^{n-k-3}+1}=E^{2^{n-k-3}+2}=\cdots=$ $E^{2^{n-k-2}}=\left\langle e_{n-k}, \ldots, e_{n}\right\rangle$ and

$$
\begin{aligned}
E^{2^{n-k-2}+1} & =E E^{2^{n-k-2}}+\cdots+E^{2^{n-k-3}} E^{2^{n-k-3}+1} \\
& =E\left\langle e_{n-k}, e_{n-k}, \ldots, e_{n}\right\rangle \subseteq\left\langle e_{n-k+1}, e_{n-k}, \ldots, e_{n}\right\rangle
\end{aligned}
$$

which contradicts $\left\langle e_{n-k}, \ldots, e_{n}\right\rangle=E^{2^{n-k-2}+1}$.

Hence this assumption is true and therefore $E^{2}=\left\langle e_{2}, \ldots, e_{n}\right\rangle$ which is also a contradiction $a_{12} \cdots a_{n-1 n}=0$.

The following example shows that there exist evolution algebras with index of nilpotency greater than $1+2^{k-3}$ and less than $1+2^{k-2}$ for all $4 \leq k \leq n$.

Example 4.8. Let $n$ and $k$ be positive integers such that $4 \leq k \leq n$. Consider an evolution algebra $E_{k}$ with basis $\left\{e_{1}, \ldots, e_{n}\right\}$ and the following multiplication table:

$$
\begin{aligned}
& e_{1} \cdot e_{1}=e_{2}+e_{3}+\cdots+e_{k}, \\
& e_{2} \cdot e_{2}=-e_{4}, \\
& e_{i} \cdot e_{i}=e_{i+1},
\end{aligned}
$$

Then one can show that $E_{k}^{3 \cdot 2^{i}}=\left\langle e_{4+i}, \ldots, e_{k}\right\rangle$ for $0 \leq i \leq k-4$ and index of nilpotency of this algebra is $1+3 \cdot 2^{k-4}$. 
Now we will consider a nilpotent evolution algebra with matrix (2.3) and a condition $\operatorname{dim} E^{2}=n-2$. Then $\operatorname{rank} A=n-2$. This implies that there are $1 \leq i \leq n-1$ and $2 \leq j \leq n$ such that $i$-th row is linear dependent to other rows and $j$-th column is linear dependent to other columns.

The following proposition presents a relation between the dimension of $E^{2}$ and $E^{3}$. In genetics, this property gives information on behavior of population of species from second and third generations.

Proposition 4.9. Let $\operatorname{dim} E^{2}=n-2$ and $i$-th row $(1 \leq i \leq n-1)$ is linear dependent to other rows and $j$-th column $(2 \leq j \leq n)$ is linear dependent to other columns. Then

$$
\operatorname{dim} E^{3}= \begin{cases}n-3 \quad \text { if } \quad & i=1 \text { or } \\ & \\ & \\ & j \neq n \text { or } \\ & i \neq 1, j \neq n, j=n-1 \text { or } j=n-2 \text { and } j \\ & j \text {-th column } \text { is zero } \\ & i \neq 1, j \neq n, j \leq n-3, i=j+1, \text { and } \\ & \\ & j \text {-th column is zero } \\ n-4 \quad \text { if } \quad & i \neq 1, j \neq n, i \neq j+1 \text { and } j \text {-th column is zero. }\end{cases}
$$

Moreover, $i=1$ implies $j=2$ and $j=n$ implies $i=n-1$.

Proof. Consider

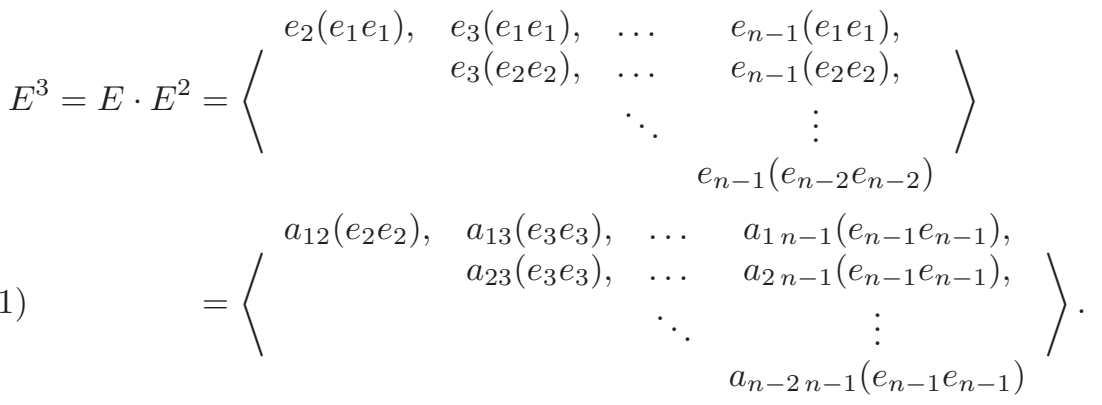

Denote by $L:=\left\langle e_{2} e_{2}, e_{3} e_{3}, \ldots, e_{n-1} e_{n-1}\right\rangle$. Obviously, $E^{3} \subseteq L$.

If $i=1$, then we obtain $a_{12}=0$ and $a_{23} \ldots a_{n-1 n} \neq 0$. Hence, $E^{2}=$ $\left\langle e_{3}, \ldots, e_{n}\right\rangle$ and $E^{3}=\left\langle e_{4}, \ldots, e_{n}\right\rangle$. Moreover, applying the same arguments as in the proof of Proposition 4.2(i) we obtain

$$
E^{2^{k-1}+1}=E^{2^{k}+2}=\cdots=E^{2^{k}}=\left\langle e_{k+2}, \ldots, e_{n}\right\rangle
$$

for all $1 \leq k \leq n-2$ and index of nilpotency for this algebra in this case is $1+2^{n-2}$

Now let $2 \leq i \leq n-1$. Then $\operatorname{dim} L=n-3$.

If $j=n$, then $a_{n-1 n}=0$ and therefore, $i=n-1$. Hence, from (4.1) one obtains $E^{3}=\left\langle e_{2} e_{2}, e_{3} e_{3}, \ldots, e_{n-2} e_{n-2}\right\rangle$. Thus, $\operatorname{dim} E^{3}=n-3$. 
Now if $j \neq n$ and $j$-th column is non-zero column, then one can easily see from (4.1) that again $E^{3}=L$. Hence, $\operatorname{dim} E^{3}=n-3$.

If $j \neq n$ and $j$-th column is zero column, then

$$
E^{3}=\left\langle\left(e_{2} e_{2}\right), \ldots,\left(e_{j} e_{j}\right),\left(e_{j+2} e_{j+2}\right), \ldots,\left(e_{n-1} e_{n-1}\right)\right\rangle .
$$

If $j=n-1$ or $j=n-2$, then $\operatorname{dim} E^{3}=n-3$.

Let $j \leq n-3$. If $i=j+1$, then $E^{3}=L$. In this case $\operatorname{dim} E^{3}=\operatorname{dim} L=n-3$.

If $i \neq j+1$, then $\operatorname{dim} E^{3}=\operatorname{dim} L-1$.

Hence, the statement of the proposition is verified.

Acknowledgments. This work is supported in part by the PAICYT, FQM143 of Junta de Andalucía (Spain). The third named author was supported by the grant NATO-Reintegration ref. CBP.EAP.RIG. 983169. The last named author would like to acknowledge ICTP OEA-AC-84 for a given support.

\section{References}

[1] J. M. Casas, M. Ladra, B. A. Omirov, and U. A. Rozikov, On evolution algebras, Algebra Colloquium. arXiv:1004.1050v1 (to appear).

[2] J. M. Casas, M. Ladra, and U. A. Rozikov, A chain of evolution, Linear Algebra Appl. 435 (2011), no. 4, 852-870.

[3] I. M. H. Etherington, Genetic algebras, Proc. Roy. Soc. Edinburgh 59 (1939), 242-258.

[4] - Duplication of linear algebras, Proc. Edinburgh Math. Soc. 2 (1941), no. 6, $222-230$.

[5] - Non-associative algebra and the symbolism of genetics, Proc. Roy. Soc. Edinburgh. Sect. B. 61 (1941), 24-42.

[6] V. Glivenkov, Algebra Mendelienne comptes rendus, (Doklady) de l'Acad. des Sci. de I'URSS 4 (1936), no. 13, 385-386 (in Russian).

[7] V. A. Kostitzin, Sur les coefficients mendeliens d'heredite, Comptes rendus de l'Acad. des Sci. 206 (1938), 883-885 (in French).

[8] Y. I. Lyubich, Mathematical Structures in Population Genetics, Biomathematics, 22, Springer-Verlag, Berlin, 1992.

[9] E. Mossel, Reconstruction on trees: beating the second eigenvalue, Ann. Appl. Probab. 11 (2001), no. 1, 285-300.

[10] A. Serebrowsky, On the properties of the Mendelian equations, Doklady A.N.SSSR 2 (1934), 33-36 (in Russian).

[11] J. P. Tian, Evolution algebras and their applications, Lecture Notes in Mathematics, 1921, Springer-Verlag, Berlin, 2008.

[12] J. P. Tian and P. Vojtechovsky, Mathematical concepts of evolution algebras in nonMendelian genetics, Quasigroups Related Systems 14 (2006), no. 1, 111-122.

L. M. CAMACHO

Dpto. Matemática Aplicada I

UNIVERSidAd DE SEVILla

Avda. Reina Mercedes, s/N. 41012, Sevilla, Spain

E-mail address: 1camacho@us.es 
1494 L. M. CAMACHO, J. R. GÓMEZ, B. A. OMIROV, AND R. M. TURDiBAEV

J. R. Gómez

UNIVERSIDAD DE SEVILla

Avda. Reina Mercedes, s/n. 41012, Sevilla, Spain

E-mail address: jrgomez@us.es

B. A. Omirov

Institute of Mathematics and Information TeChnologies

Do'RMON Yo'Li STR., 29, 100125, TASHKENT, UZBEKISTAN

E-mail address: omirovb@mail.ru

R. M. TURDIBAEV

Department of Mathematics

NATIONAL UNIVERSity OF UZBEKISTAN

Vuzgorogok, 27, 100174, TASHKEnt, UzBEKISTAN

E-mail address: rustamtm@yahoo.com 